\title{
Clinical Characteristics of Chronic Lung Abscess Associated with Parvimonas micra Diagnosed Using Metagenomic Next-Generation Sequencing
}

This article was published in the following Dove Press journal: Infection and Drug Resistance

\author{
Yaping Zhang ${ }^{1,2, *}$ \\ Ping Song ${ }^{1,3, *}$ \\ Ruhui Zhang' \\ Yake Yao' \\ Lisha Shen ${ }^{\prime}$ \\ Qiang $\mathrm{Ma}^{4}$ \\ Jianying Zhou (D) \\ Hua Zhou (iD) \\ 'Department of Respiratory and Critical \\ Care Medicine, The First Affiliated \\ Hospital, Zhejiang University School of \\ Medicine, Hangzhou, Zhejiang, 310003, \\ People's Republic of China; ${ }^{2}$ Department \\ of Respiratory and Critical Care \\ Medicine, Shangyu People's Hospital, \\ Shaoxing, Zhejiang, 312300, People's \\ Republic of China; ${ }^{3}$ Department of \\ Critical Care Medicine, Deqing People's \\ Hospital, Huzhou, Zhejiang, 3/3200, \\ People's Republic of China; ${ }^{4}$ Department \\ of Respiratory Diseases, Yuhang Second \\ People's Hospital, Hangzhou, Zhejiang, \\ 3 I I 100, People's Republic of China
}

*These authors contributed equally to this work
Correspondence: Hua Zhou; jianying Zhou Department of Respiratory and Critical Care Medicine, The First Affiliated Hospital, Zhejiang University School of Medicine, Hangzhou, Zhejiang, 310003, People's Republic of China

Email zhouhual@zju.edu.cn; zjyhz@zju.edu.cn
Purpose: Parvimonas micra (P. micra) is a Gram-positive anaerobic bacterium distributed in the oral cavity, with a potential to become pathogenic causing lung abscess. Due to the lack of specificity of symptoms and the difficulty in culture, the diagnosis of lung abscess associated with P. micra is delayed. It is essential to elucidate the clinical characteristics of lung abscess associated with P. micra.

Methods: From January 2019 to July 2020, five patients with chronic lung abscess associated with $P$. micra diagnosed by pathological biopsy and metagenomic next-generation sequencing (mNGS) were analyzed in this retrospective study.

Results: Among the five patients, four had a history of smoking, three had periodontitis, and two had a history of drinking. The average course of the disease was 6.5 months. Highdensity flake-like or mass shadows with irregular boundaries were observed in the chest computed tomography (CT) images of the five patients, and liquefactive necrosis was detected in the middle of the lesions; however, no gas-liquid plane or cavity was noted, making it difficult to distinguish a lung cancer. The pathological biopsy of the five patients showed chronic inflammation of lung tissue, and P. micra was detected by mNGS in the biopsy or bronchoalveolar lavage fluid samples. Two patients were treated with amoxicillinclavulanate, two had metronidazole, and one had moxifloxacin. Among them, four recovered after receiving antibiotic treatment, and the remaining one underwent surgical resection due to poor antibiotic treatment effect.

Conclusion: Chronic lung abscess associated with P. micra, common in elderly male smokers with poor oral hygiene, is often diagnosed in a delayed manner and misdiagnosed as lung cancer. The mNGS technology is beneficial to the rapid determination of P. micra. Keywords: Parvimonas micra, P. micra, lung abscess, metagenomic next-generation sequencing, mNGS

\section{Introduction}

Parvimonas micra (P. micra) is a Gram-positive anaerobic bacterium with a diameter of $0.3-0.7 \mu \mathrm{m}$ and grows slowly. It is arranged in pairs or chains and can be commonly found in the human oral cavity. ${ }^{1-3} P$. micra causes bacteremia, rachitis, arthritis, infective endocarditis, liver abscess, renal abscess, brain abscess, pleural effusion, and lung abscess. ${ }^{4-13}$ A lung abscess is a suppurative lesion of the lung tissue caused by various pathogenic bacteria. The bacteria causing the infection are consistent with the common bacteria in the oral cavity and the upper respiratory tract. ${ }^{14} \mathrm{~A}$ lung abscess is defined as chronic if the course of the disease 
lasts $>3$ months. However, due to the difficulty in cultivating anaerobic bacteria, P. micra has been rarely identified as the pathogenic bacterium of lung abscess, and only slight data are available on the clinical characteristics of the lung abscess associated with P. micra. The infections caused by anaerobic bacteria, including $P$. micra, can be identified using microbial detection technology based on nucleic acid detection, such as the metagenomic next-generation sequencing (mNGS). In this study, the clinical characteristics of chronic lung abscess associated with $P$. micra were analyzed through a retrospective analysis of five patients who received treatment at the First Affiliated Hospital of Zhejiang University School of Medicine from January 2019 to July 2020. Although these five patients could not be diagnosed definitively for a long time, they were later diagnosed with chronic lung abscess associated with P. micra through mNGS detection.

\section{Patients and Methods}

\section{Patient Enrollment Research Design and Enrolled Patients}

This retrospective study was conducted at the First Affiliated Hospital of Zhejiang University School of Medicine. The bronchoalveolar lavage fluid (BALF) or lung biopsy tissue specimens of patients with chronic lung abscess, admitted to the hospital from January 2019 to July 2020 , were detected by mNGS. The detection results suggested P. micra was the pathogenic bacterium, which was consistent with the clinical evidence, indicating that the chronic lung abscess was associated with P. micra. In this study, five patients with chronic lung abscess associated with $P$. micra were enrolled. The information collected included patients' age, gender, underlying disease, clinical manifestation, disease progression, laboratory and imaging examination results, molecular biological diagnosis, antibacterial scheme, treatment, and prognosis.

\section{mNGS Testing}

Samples were collected and immediately transported on the dry ice to a commercial laboratory (IngeniGen XunMinKang Laboratory, Hangzhou) for mNGS testing within 4 hours after collection. Briefly, DNA was extracted from $300 \mu \mathrm{L}$ of BAL and the sequencing library was prepared using the Total Nucleic Acid Extraction Kit and the mNGS-DNA Library Prep Kit (IngeniGen XunMinKang Biotechnology, Zhejiang, China). Sequencing was performed using the 75-bp pairedend protocol on the Illumina Nextseq550 platform. At least
2.5 million reads ( $75 \mathrm{bp})$ were obtained from each sample after sequencing. The sequence data was analyzed by IngeniSeq$\mathrm{MG}^{\circledR}$ v1.0 mNGS software (IngeniGen XunMinKang Biotechnology, Zhejiang, China) which contains a proprietary curated database consisting of more than 20,000 microbial reference genomes. The detailed methods regarding the wetlab and bioinformatics had been described previously. ${ }^{15}$

\section{Results}

\section{General Characteristics}

The five patients were all males, aged between 46 and 82 years, with a body mass index (BMI) of 18-23 and community-onset. Four patients had a long history of smoking, three were complicated with periodontitis, and two had a history of drinking. None of the 5 patients had the following common risk factors for pulmonary abscess: HIV infection, diabetes, tracheotomy, chronic sinusitis, and so on. None of the five patients administered any immunosuppressive drugs, such as glucocorticoids. Three patients presented complications, but none of them had any extrapulmonary infection (empyema, brain abscess, endocarditis, skin and soft tissue infection, abdominal infection, etc). The average time taken from the clinical onset to definitive diagnosis of the five patients was 6.5 (range: 2 8.5) months (Table 1).

\section{Clinical Manifestations of the Onset}

The five patients had cough and expectoration at the time of onset; however, the sputum had no odor, but three of the patients had blood-stained sputum. Two patients had a fever with a maximum body temperature of $39^{\circ} \mathrm{C}$, while two cases had chest pain, and two cases showed dyspnea.

\section{Diagnosis and Treatment at Early Onset}

The five patients were initially diagnosed with pneumonia, and all received antibiotic treatment for 1 2.5 months. Four patients received fluoroquinolone (moxifloxacin or levofloxacin) treatment, including two cases treated with fluoroquinolone monotherapy and two with the combination therapy with cefdinir. The remaining one case was treated with cefdinir monotherapy (Table 1).

\section{Changes in Clinical Manifestations After Initial Treatment}

After the initial diagnosis, four patients received antibiotic treatment, and the symptoms improved significantly; among them, symptoms disappeared in three cases, and one case had a slight cough. The remaining one patient had cough, 


\begin{tabular}{|c|c|c|c|c|c|c|}
\hline & 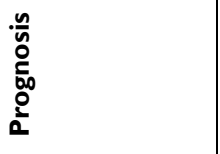 & Uे & U. & गै & 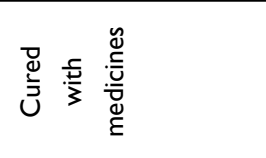 & 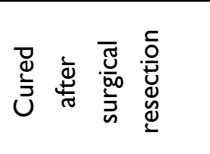 \\
\hline & 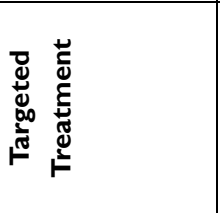 & 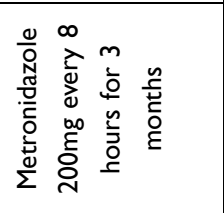 & 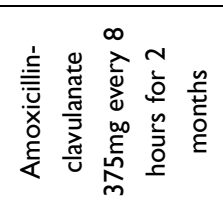 & 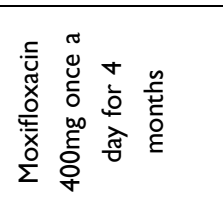 & 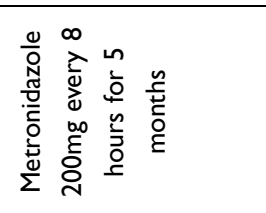 & 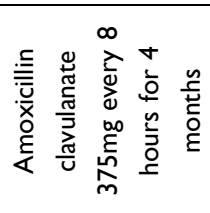 \\
\hline & 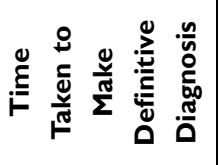 & 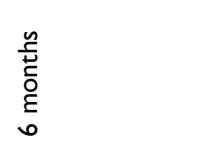 & 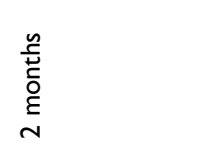 & 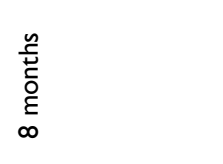 & 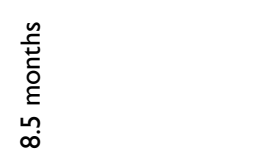 & 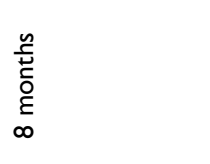 \\
\hline & 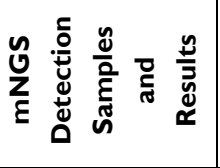 & 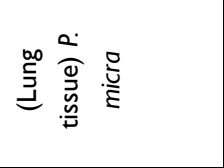 & 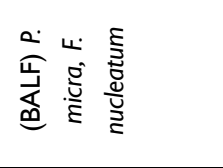 & 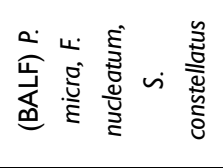 & 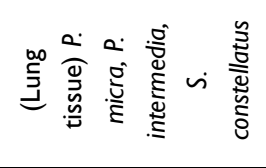 & 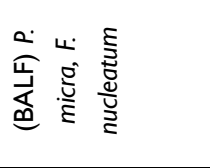 \\
\hline & 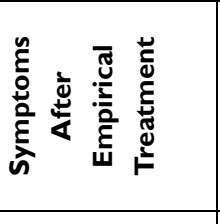 & $\begin{array}{l}0 \\
\stackrel{0}{0} \\
z\end{array}$ & $\begin{array}{l}\text { ¿̊ } \\
\text { Zे }\end{array}$ & 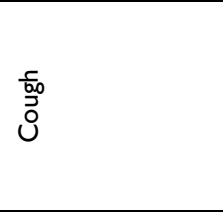 & $\begin{array}{l}\stackrel{0}{0} \\
\stackrel{0}{z}\end{array}$ & 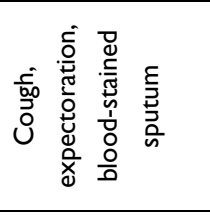 \\
\hline & 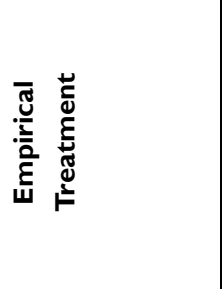 & 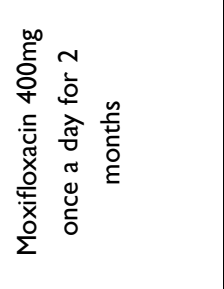 & 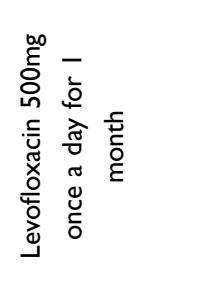 & 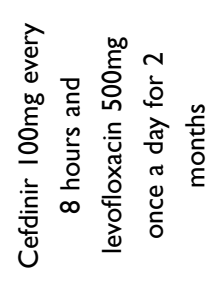 & 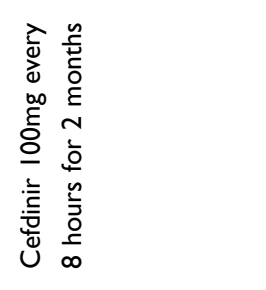 & 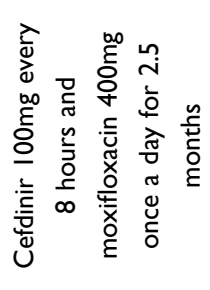 \\
\hline & 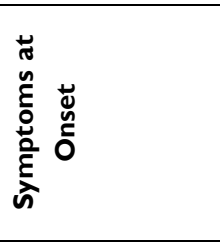 & 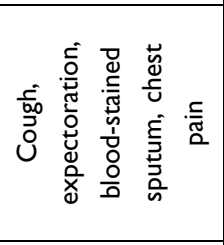 & 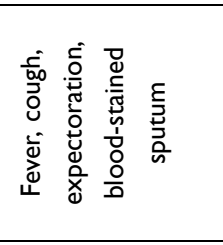 & 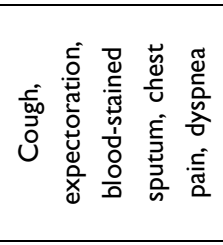 & 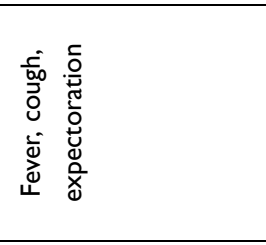 & 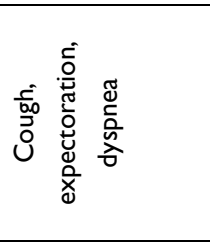 \\
\hline & 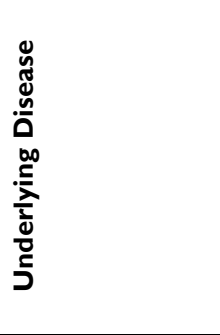 & $\begin{array}{l}\stackrel{0}{0} \\
\text { Z }\end{array}$ & $\begin{array}{l}\stackrel{0}{ \pm} \\
\text { z }\end{array}$ & 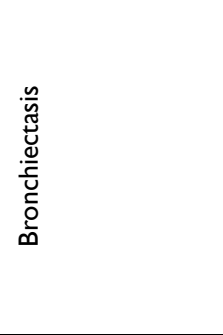 & 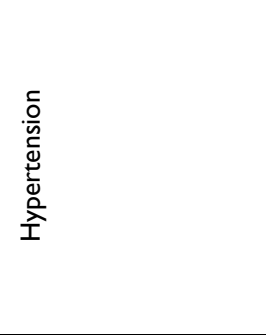 & 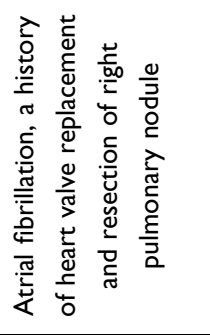 \\
\hline & 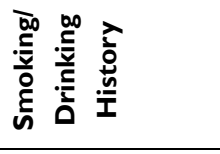 & 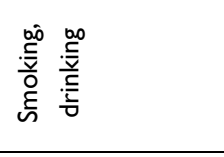 & 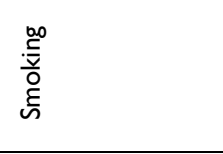 & $\begin{array}{l}\stackrel{0}{0} \\
\text { Zे }\end{array}$ & 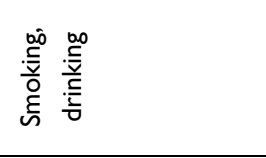 & 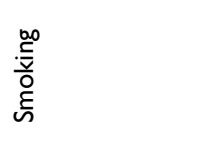 \\
\hline & 品 & $\overline{6}$ & $\bar{\infty}$ & $\mathscr{f}$ & $\widetilde{\infty}$ & ธ్ \\
\hline & 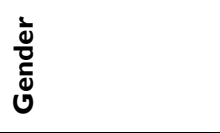 & $\frac{\frac{\omega}{\pi}}{\Sigma}$ & $\frac{\frac{\omega}{\pi}}{\sum}$ & $\frac{\frac{\omega}{N}}{\Sigma}$ & $\frac{\frac{\omega}{\pi}}{\Sigma}$ & $\frac{\frac{\omega}{\sigma}}{\Sigma}$ \\
\hline & $\dot{\mathbf{z}}$ & - & $N$ & $m$ & $\sigma$ & in \\
\hline
\end{tabular}


expectoration, and blood-stained sputum after medical treatment (Table 1). About 2 months after the initial treatment, no improvement was seen in the lung computed tomography (CT) scan of the five patients; four patients were suspected of lung cancer, and one case was misdiagnosed with cryptogenic organizing pneumonia. Because of the remission of clinical symptoms 1 2.5 months after the initial treatment, all patients stopped taking medicine and were under observation. The delay from the end of initial antibiotic treatment to diagnosis was $1 \sim 6.5$ months.

\section{Imaging Manifestations of Lung CT}

The locations of lung abscesses in the five patients were as follows: two cases in the upper lobe of left lung and one case in the lower lobe of left lung, upper lobe of right lung, and middle lobe of right lung, respectively. Among the initial imaging manifestations of the patients, three cases had lumpy shadows with unclear boundaries with one had small cavities in the lesion, and two cases had lumpy shadows with clear boundaries. After the progression of the disease, it was difficult to distinguish from lung tumor based on the imaging manifestations of the five patients. The necrosis of the local lung tissue was detected within the lesions of the patients: two cases had small cavities in the lung, no typical gas-liquid pyogenic cavity was observed in all the patients, and one case developed occlusion in the left inferior lobar bronchus. Detailed lung images of two patients are shown in Figures 1 and 2.

\section{Bronchoscopy, Lung Biopsy, and Pathology}

Since distinguishing the disease from lung cancer through imaging is challenging, all five patients underwent bronchoscopy and CT-guided percutaneous lung biopsy. The bronchoalveolar lavage was performed at the infected site under a bronchoscope, but no purulent secretion was observed in the bronchoalveolar lavage fluid (BALF). Four patients underwent lung puncture biopsy, including repeated lung puncture biopsy in one patient, and the remaining one patient underwent the endobronchial ultrasound-guided transbronchial needle aspiration (EBUSTBNA). The biopsy showed inflammation of the lung tissue and purulent changes in some cases. The pathological results of these patients are described in Table 2 .

\section{Laboratory Examination}

At the time of admission, the mean white blood cell level of the five patients was $12.6 \times 10^{9} / \mathrm{L}$ (range: $6-31.7$ ), the mean C-reactive protein (CRP) level was $60.2 \mathrm{mg} / \mathrm{L}$

\section{Time point 1}
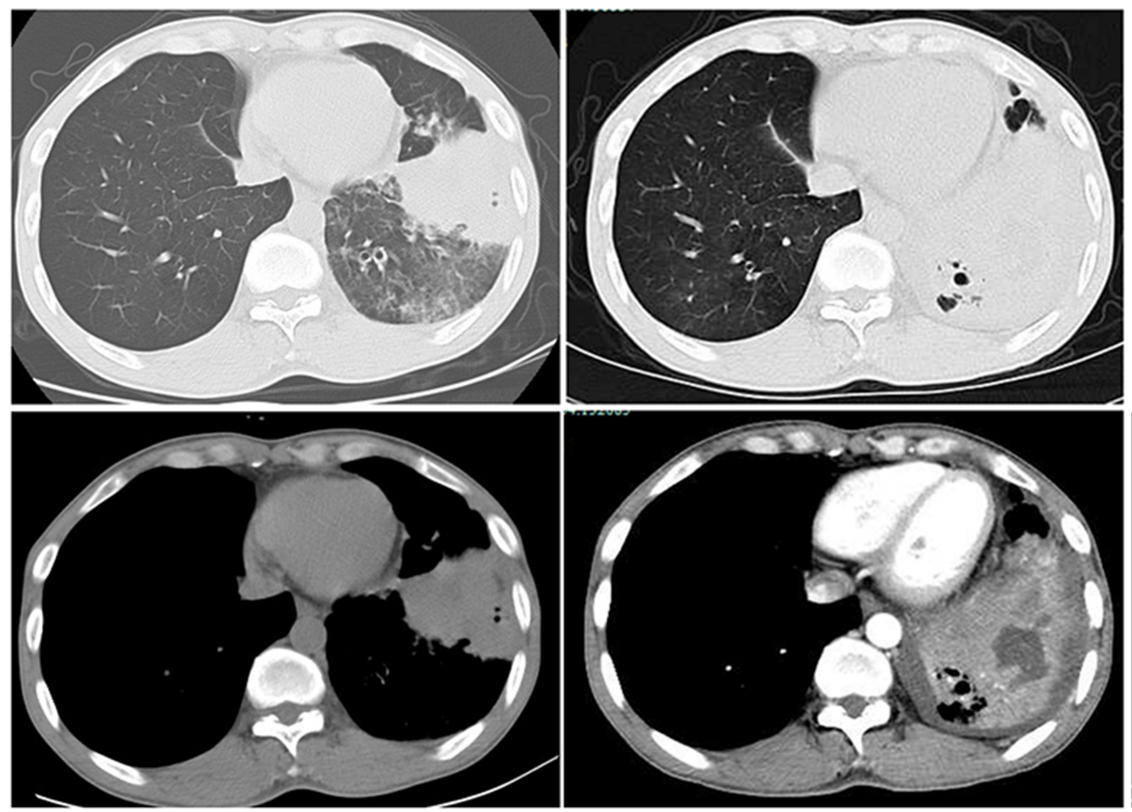

Time point 2

Time point 3
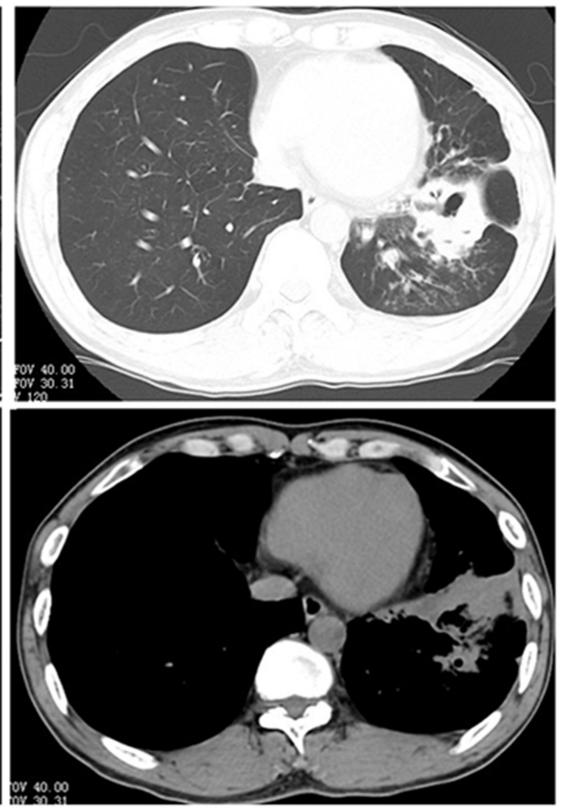

Figure I Lung CT manifestation of case 3. Time point I: At the first visit, clump-like lung consolidation was visible in the left lower lobe, accompanied by the exudation of surrounding ground-glass lesion, and central necrosis was not obvious. Time point 2: At 8 months of onset, large areas of lung consolidation were visible with liquefactive necrosis and small cavities in the lesion. Time point 3: After effective treatment for 3 months, the lesion was significantly smaller with residual cavities and fibrous proliferation lesions. 


\section{Time point 1}
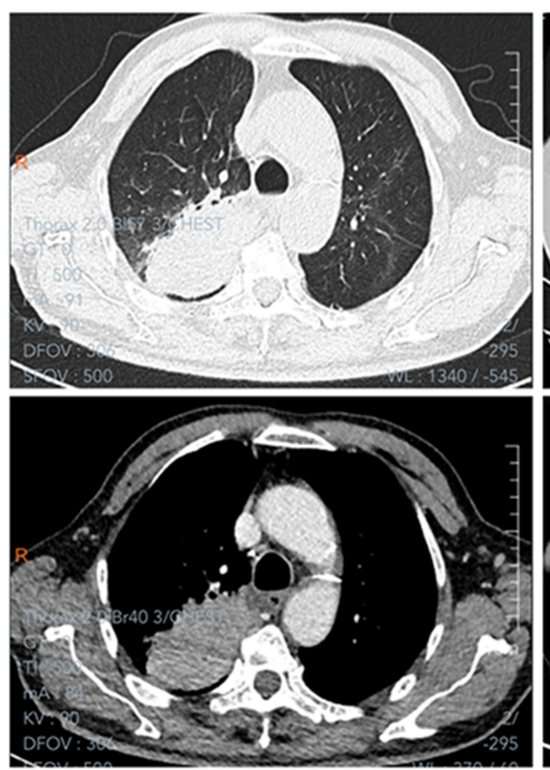

Time point 2

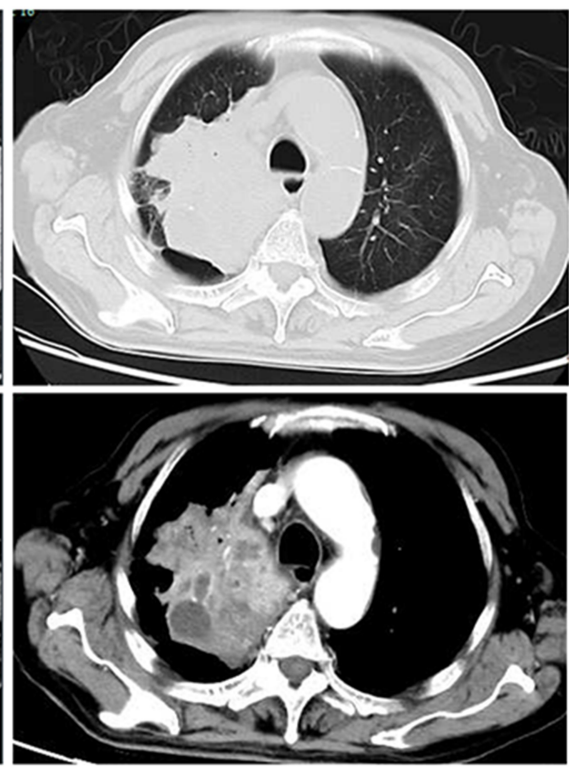

Time point 3

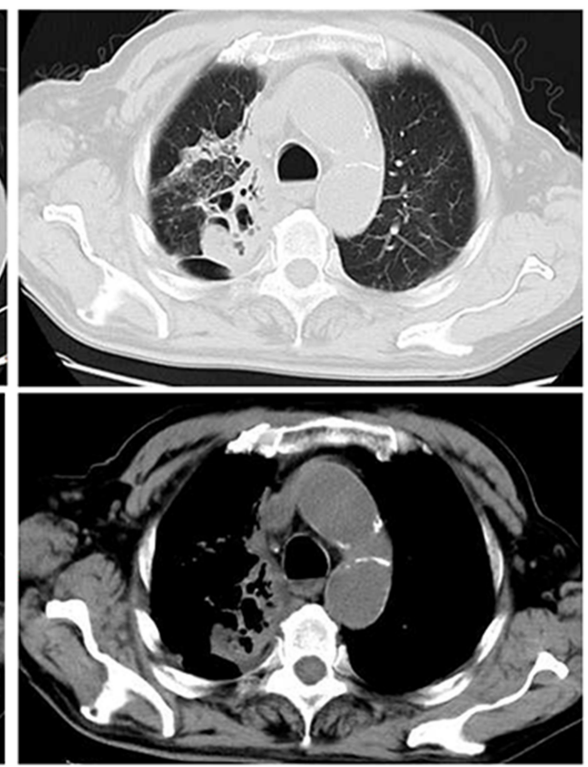

Figure 2 Lung CT manifestation of case 4. Time point I: At the first visit, a mass lung consolidation shadow was visible in the right upper lobe. Time point 2: At 8 months of onset, a notably enlarged mass is visible, with liquefaction necrosis in the middle of the lesion. Time point 3: After effective treatment for 3 months, the lesion was significantly smaller with residual cavities and fibrous lesions.

(range: 7-127.1), the mean albumin level was $36.2 \mathrm{~g} / \mathrm{L}$ (range: 32.7-45.8), the mean hemoglobin level was $118.6 \mathrm{~g} / \mathrm{L}$ (range: $94-143$ ), and the mean red blood cell level was $4.06 \times 10^{12} / \mathrm{L}$ (range: $3.25-4.85$ ). One case had increased procalcitonin level, which was $2.18 \mathrm{ng} / \mathrm{mL}$ (normal range: $0-0.5 \mathrm{ng} / \mathrm{mL}$ ).

\section{Diagnosis of Pathogen}

Blood culture and sputum culture were performed in these five patients, but the results were all negative. All five patients underwent bronchoscopy or percutaneous lung puncture biopsy. The BALF or lung tissue was collected and sent for routine bacterial smear and culture, followed

Table 2 Lung Biopsy Pathology of Five Patients with Chronic Lung Abscess Associated with P. micra

\begin{tabular}{|c|c|c|c|}
\hline No. & Lesion Site & Biopsy Method & Lung Biopsy Pathology \\
\hline I & $\begin{array}{l}\text { Left upper } \\
\text { lung lobe }\end{array}$ & CT-guided percutaneous lung puncture & Chronic suppurative inflammation of lung tissue \\
\hline 2 & $\begin{array}{l}\text { Right middle } \\
\text { lung lobe }\end{array}$ & $\begin{array}{l}\text { Endobronchial ultrasound-guided } \\
\text { transbronchial needle aspiration }\end{array}$ & Chronic inflammation of lung with cryptogenic organizing pneumonia \\
\hline 3 & $\begin{array}{l}\text { Left lower } \\
\text { lung lobe }\end{array}$ & CT-guided percutaneous lung puncture & Chronic inflammation of lung tissue with fibrous hyperplasia \\
\hline 4 & $\begin{array}{l}\text { Right upper } \\
\text { lung lobe }\end{array}$ & CT-guided percutaneous lung puncture & $\begin{array}{l}\text { I. (At early onset) lung tissue, with fibrous tissue hyperplasia in the alveolar } \\
\text { cavity; } \\
\text { 2. (After } 8.5 \text { months) lung tissue, interstitial fibrous tissue hyperplasia with } \\
\text { multiple inflammatory cell infiltration }\end{array}$ \\
\hline 5 & $\begin{array}{l}\text { Left upper } \\
\text { lung lobe }\end{array}$ & $\begin{array}{l}\text { I. CT-guided percutaneous lung } \\
\text { puncture } \\
\text { 2. Surgical resection }\end{array}$ & $\begin{array}{l}\text { I. Chronic inflammation with fibrous tissue hyperplasia and infiltration of the } \\
\text { lymph, plasma cells, and eosinophils, regional necrosis } \\
\text { 2. Bronchogenic cyst with chronic suppurative inflammation and the formation of } \\
\text { a multifocal abscess }\end{array}$ \\
\hline
\end{tabular}

Abbreviation: $\mathrm{CT}$, computed tomography. 
by mNGS. In the BALF smears, white blood cells were detected in all five patients, gram-positive cocci were detected in three cases. In the BALF culture, Streptococcus constellatus was identified in one case, no fungi and Mycobacterium were identified. The BALF of three patients and the lung puncture tissue specimens of the remaining two patients were sent for mNGS analysis. After the mNGS detection, one patient was diagnosed with simple $P$. micra infection, two cases with co-infection of $P$. micra and Fusobacterium nucleatum, one case with coinfection of P. micra, F. nucleatum, and S. constellatus, and one case with co-infection of P. micra, Prevotella intermedia, and S. constellatus (Table 1).

\section{Targeted Treatment Regimen}

After a definitive diagnosis, the patients received the following antibiotic treatment: amoxicillin and clavulanate potassium for two cases, metronidazole for two cases, and moxifloxacin for one case (Table 1).

\section{Prognosis}

The prognosis of the five patients was satisfactory. The antibiotic treatment on the four patients achieved good effects, the clinical symptoms improved, and chest CT showed that the lesions were completely absorbed. The antibiotic treatment on the remaining one patient failed, and no obvious improvement was seen in the symptoms. A chest imaging re-examination showed that the lesion was enlarged, which was removed surgically. The postoperative pathology revealed a bronchial cyst with chronic suppurative inflammation and the formation of a multifocal abscess (Table 1).

\section{Discussion}

P. micra is a microorganism commonly found in the oral cavity. Reportedly, the level of P. micra and other bacteria in smokers' oral cavity is higher than that of nonsmokers. ${ }^{16-18}$ In this study, we found that most of the patients with chronic lung abscess associated with $P$. micra had a long history of smoking. Hence, we speculated that long-term smoking might increase the risk of chronic lung abscess associated with $P$. micra.

Since there was no specificity in the symptoms of these patients, and we did not have sufficient knowledge of the clinical characteristics of chronic lung abscess associated with $P$. micra, we might not have analyzed the pathogenic bacteria at the beginning of the disease. This phenomenon resulted in prolonged diagnosis and treatment of the disease and further led to chronic lung abscess. P. micra can enhance inflammation, especially persistent inflammation by stimulating the release of some inflammatory transmitters by human macrophages. ${ }^{19}$ The imaging manifestations of the five patients in this study made it difficult to distinguish their disease from lung cancer; a definitive diagnosis requires lung biopsy and mNGS detection. Guo et al reported a patient who underwent a lung puncture biopsy because of a suspected lung tumor, while the next-generation sequencing technology confirmed that the chronic lung abscess associated with $P$. micra and $F$. nucleatum. ${ }^{13}$ Gorospe et al reported a patient who underwent two bronchoscopy biopsies and two CT-guided lung puncture biopsies because of suspected lung cancer, which was ultimately confirmed to be lung consolidation attributable to $P$. micra infection. ${ }^{20}$ Both cases were similar to our cases.

Previous studies discovered that $P$. micra is often sensitive to metronidazole, penicillin, amoxicillin-clavulanate, and clindamycin. ${ }^{1,21}$ The initial administration of fluoroquinolone or cefdinir did not affect the patients in this study, and a significant efficacy was achieved after the treatment with metronidazole amoxicillin-clavulanate and other antibiotics casting sensitivity to anaerobes. This finding might be associated with the resistance of Gram-positive anaerobic cocci (GPAC) to fluoroquinolones. ${ }^{22}$ However, in one case in this study, the initial treatment using levofloxacin failed, and after a definitive diagnosis, the treatment with moxifloxacin was effective, suggesting that moxifloxacin had a better treatment effect than levofloxacin. As a result, we should search for pathogens at the earliest for a definitive diagnosis, select antibiotics treatment causing sensitivity to the identified pathogens, and achieve the transition from empirical treatment to targeted treatment.

This study showed that the co-infection of $P$. micra with other oral symbiotic bacteria was simple and that chronic lung abscesses associated with $P$. micra infection required a prolonged duration for treatment with antibiotics due to a poor effect. In this study, most of the cases with chronic lung abscess associated with $P$. micra were successfully treated with antibiotics, and one patient with chronic lung abscess associated with the co-infection of P. micra and F. nucleatum had the lesion removed surgically due to the poor antibiotic treatment effect. Didilescu et al found a synergy between $P$. micra and $F$. nucleatum in the oral infection. ${ }^{23}$ Thus, we speculated that both bacteria might also affect chronic lung abscess and enhance the pathogenicity. Özok et al demonstrated that the resistance of the dual-species biofilms 
formed by $P$. micra and $F$. nucleatum to antimicrobial agents was stronger than the monospecies biofilms formed by the two bacteria. ${ }^{24}$ These findings were consistent with the prognosis of the case in this study.

mNGS is a novel clinical metagenomics technology that does not require culture and is based on sequencing technology. By analyzing the content and abundance of DNA or the RNA of microorganisms in clinical samples, this technology could be used to determine the pathogenic bacteria and was successfully applied to identify the pathogens from patients' BALF or lung tissue samples. ${ }^{25-28}$ Importantly, the sensitivity of using mNGS to diagnose infectious diseases was higher than conventional culture, especially for bacteria that are difficult to cultivate, such as anaerobes. ${ }^{29,30}$ Moreover, mNGS is less affected by antibiotic exposure before sample detection, ${ }^{29}$ and thus has more advantages in detecting the co-infection of multiple bacteria. $^{30}$

Nevertheless, the present study has some limitations. First, this is a retrospective study with small sample size. Second, we failed to perform an anaerobic culture of the lung specimens routinely. Also, the mNGS results were not validated. Third, some cases of this study were coinfections of $P$. micra and other oral symbiotic bacteria. Thus, additional studies are required for the differences between co-infection and single bacterial infection and their respective clinical characteristics.

Together, we summarized the clinical characteristics of patients with chronic lung abscess associated with $P$. micra as follows: the majority of such patients have a long history of smoking, their disease course is long, the symptoms are not typical, the chest imaging manifestations did not show liquid-gas plane and cavity of typical lung abscess, and the disease could not be diagnosed in time, resulting in the formation of chronic infection and abscess; these often led to the misdiagnosis of lung cancer. A good prognosis can be achieved by timely and accurate pathology, early identification of pathogens, and effective antibiotic treatment. The mNGS technology is beneficial to the rapid determination of pathogens.

\section{Ethics Approval and Consent for Publication}

This study was conducted in accordance with the Declaration of Helsinki and had been reviewed and approved by the Research Ethics Committee of the First Affiliated Hospital of Zhejiang University (IIT2021049A).
The patients provided written informed consent for the publication of the clinical information including lung CT images.

\section{Author Contributions}

All authors made a significant contribution to the work reported, whether that is in the conception, study design, execution, acquisition of data, analysis and interpretation, or in all these areas; took part in drafting, revising or critically reviewing the article; gave final approval of the version to be published; have agreed on the journal to which the article has been submitted; and agree to be accountable for all aspects of the work.

\section{Funding}

This study was supported by a research grant from the National Natural Science Foundation of China (81971897) and the Natural Science Foundation of Zhejiang Province (LQ20H010006). The funders had no role in the study design, data collection and analysis, decision to publish, or preparation of the manuscript.

\section{Disclosure}

The authors declare that they have no conflicts of interest.

\section{References}

1. Boattini M, Bianco G, Cavallo R, Costa C. Parvimonas micra bacteremia following endoscopic retrograde cholangiopancreatography: a new route of infection. Anaerobe. 2018;54:136-139. doi:10.1016/j. anaerobe.2018.09.003

2. Tindall BJ, Euzéby JP. Proposal of Parvimonas gen. nov. and Quatrionicoccus gen. nov. as replacements for the illegitimate, prokaryotic, generic names Micromonas Murdoch and Shah 2000 and Quadricoccus Maszenan et al. 2002, respectively. Int J Syst Evol Microbiol. 2006;56(Pt 11):2711-2713. doi:10.1099/ijs.0.64338-0

3. Watanabe T, Hara Y, Yoshimi Y, Fujita Y, Yokoe M, Noguchi Y. Clinical characteristics of bloodstream infection by Parvimonas micra: retrospective case series and literature review. BMC Infect Dis. 2020;20(1):578. doi:10.1186/s12879-020-05305-y

4. Badri M, Nilson B, Ragnarsson S, Senneby E, Rasmussen M. Clinical and microbiological features of bacteraemia with Gram-positive anaerobic cocci: a population-based retrospective study. Clin Microbiol Infect. 2019;25(6):760.e1-.e6. doi:10.1016/j.cmi.2018.09.001

5. Jones SL, Riordan JW, Glasgow AL, Botes J, Boutlis CS. Two cases of spondylodiscitis caused by Parvimonas micra. Intern Med J. 2015;45 (10):1090-1091. doi:10.1111/imj.12877

6. Dietvorst M, Roerdink R, Leenders A, Kiel MA, Bom LPA. Acute mono-arthritis of the knee: a case report of infection with Parvimonas micra and concomitant pseudogout. J Bone Jt Infect. 2016;1(1):65-67. doi:10.7150/jbji.16124

7. Sultan AA, Cantrell WA, Khlopas A, et al. Acute septic arthritis of the knee: a rare case report of infection with Parvimonas micra after an intra-articular corticosteroid injection for osteoarthritis. Anaerobe. 2018;51:17-20. doi:10.1016/j.anaerobe.2017.12.015 
8. Gomez CA, Gerber DA, Zambrano E, Banaei N, Deresinski S, Blackburn BG. First case of infectious endocarditis caused by Parvimonas micra. Anaerobe. 2015;36:53-55. doi:10.1016/j. anaerobe.2015.10.007

9. Chaucer B, Smith N, Beatty D, Yadav M. Multiple hepatic abscess from Parvimonas micra: an emerging gastrointestinal microbe. $A C G$ Case Rep J. 2018;5:e70. doi:10.14309/crj.2018.70

10. Garrido-Jareño M, Frasquet-Artes J, Tasias-Pitarch M, LópezHontangas JL. First case of renal abscess by Parvimonas micra. Enferm Infecc Microbiol Clin. 2019;37(2):140-141. doi:10.1016/j. eimc.2018.03.002

11. Ochi F, Tauchi H, Miyata T, et al. Brain abscess associated with polymicrobial infection after intraoral laceration: a pediatric case report. Case Rep Pediatr. 2020;2020:8304302. doi:10.1155/2020/ 8304302

12. Cobo F, Rodríguez-Granger J, Sampedro A, Aliaga-Martínez L, Navarro-Marí JM. Pleural effusion due to Parvimonas micra. A case report and a literature review of 30 cases. Rev Esp Quimioter. 2017;30(4):285-292.

13. Guo W, Gao B, Li L, et al. A community-acquired lung abscess attributable to odontogenic flora. Infect Drug Resist. 2019;12:24672470. doi:10.2147/IDR.S218921

14. Allewelt M, Lode H. [Diagnosis and therapy of abscess forming pneumonia]. Ther Umsch. 2001;58(10):599-603. German. doi:10.1024/0040-5930.58.10.599

15. Fang X, Xu M, Fang Q, et al. Real-time utilization of metagenomic sequencing in the diagnosis and treatment monitoring of an invasive adenovirus B55 infection and subsequent herpes simplex virus encephalitis in an immunocompetent young adult. Open Forum Infect Dis. 2018;5(6):ofy114. doi:10.1093/ofid/ofy114

16. Delima SL, McBride RK, Preshaw PM, Heasman PA, Kumar PS. Response of subgingival bacteria to smoking cessation. J Clin Microbiol. 2010;48(7):2344-2349. doi:10.1128/JCM.01821-09

17. Shchipkova AY, Nagaraja HN, Kumar PS. Subgingival microbial profiles of smokers with periodontitis. J Dent Res. 2010;89 (11):1247-1253. doi:10.1177/0022034510377203

18. Ota-Tsuzuki C, Brunheira AT, Mayer MP. 16S rRNA region based PCR protocol for identification and subtyping of Parvimonas micra. Braz J Microbiol. 2008;39(4):605-607. doi:10.1590/S151783822008000400001

19. Yoshioka M, Grenier D, Mayrand D. Binding of Actinobacillus actinomycetemcomitans lipopolysaccharides to Peptostreptococcus micros stimulates tumor necrosis factor alpha production by macrophage-like cells. Oral Microbiol Immunol. 2005;20(2):118-121. doi:10.1111/j.1399-302X.2004.00204.x
20. Gorospe L, Bermudez-Coronel-Prats I, Gomez-Barbosa CF, OlmedoGarcia ME, Ruedas-Lopez A, Gomez Del Olmo V. Parvimonas micra chest wall abscess following transthoracic lung needle biopsy. Korean J Intern Med. 2014;29(6):834-837. doi:10.3904/ kjim.2014.29.6.834

21. Lee Y, Park Y, Kim MS, et al. Antimicrobial susceptibility patterns for recent clinical isolates of anaerobic bacteria in South Korea. Antimicrob Agents Chemother. 2010;54(9):3993-3997. doi:10.1128/ AAC.00481-10

22. Shilnikova II, Dmitrieva NV. Evaluation of antibiotic susceptibility of Gram-Positive Anaerobic Cocci isolated from cancer patients of the N. N. Blokhin Russian Cancer Research Center. J Pathog. 2015;2015:648134. doi:10.1155/2015/648134

23. Didilescu AC, Rusu D, Anghel A, et al. Investigation of six selected bacterial species in endo-periodontal lesions. Int Endod J. 2012;45 (3):282-293. doi:10.1111/j.1365-2591.2011.01974.x

24. Özok AR, Wu MK, Luppens SB, Wesselink PR. Comparison of growth and susceptibility to sodium hypochlorite of mono- and dual-species biofilms of Fusobacterium nucleatum and Peptostreptococcus (micromonas) micros. J Endod. 2007;33(7):819822. doi:10.1016/j.joen.2007.03.008

25. Li Y, Sun B, Tang X, et al. Application of metagenomic next-generation sequencing for bronchoalveolar lavage diagnostics in critically ill patients. Eur J Clin Microbiol Infect Dis. 2020;39(2):369374. doi:10.1007/s10096-019-03734-5

26. Li H, Gao H, Meng H, et al. Detection of pulmonary infectious pathogens from lung biopsy tissues by metagenomic next-generation sequencing. Front Cell Infect Microbiol. 2018;8:205. doi:10.3389/ fcimb.2018.00205

27. Li G, Huang J, Li Y, Feng J. The value of combined radial endobronchial ultrasound-guided transbronchial lung biopsy and metagenomic next-generation sequencing for peripheral pulmonary infectious lesions. Can Respir J. 2020;2020:2367505. doi:10.1155/ 2020/2367505

28. Chiu CY, Miller SA. Clinical metagenomics. Nat Rev Genet. 2019;20 (6):341-355. doi:10.1038/s41576-019-0113-7

29. Miao Q, Ma Y, Wang Q, et al. Microbiological diagnostic performance of metagenomic next-generation sequencing when applied to clinical practice. Clin Infect Dis. 2018;67(suppl_2):S231-S40. doi:10.1093/cid/ciy693

30. Wang J, Han Y, Feng J. Metagenomic next-generation sequencing for mixed pulmonary infection diagnosis. BMC Pulm Med. 2019;19 (1):252. doi:10.1186/s12890-019-1022-4
Infection and Drug Resistance

\section{Publish your work in this journal}

Infection and Drug Resistance is an international, peer-reviewed openaccess journal that focuses on the optimal treatment of infection (bacterial, fungal and viral) and the development and institution of preventive strategies to minimize the development and spread of resistance. The journal is specifically concerned with the epidemiology of antibiotic resistance and the mechanisms of resistance development and diffusion in both hospitals and the community. The manuscript management system is completely online and includes a very quick and fair peerreview system, which is all easy to use. Visit http://www.dovepress.com/ testimonials.php to read real quotes from published authors. 